\title{
OPTIMIZATION OF ASYMMETRIC MAGNETIC RECORDING HEADS INCLINATION ANGLE AND STUDYING THE CHARACTERISTICS FOR A HIGHER BIT DENSITY RECORDING OF HDD
}

\author{
Ammar Isam Edress \\ Geology of Petroleum and Sediments Department, Technical College of Petroleum and Minerals, Duhok Polytechnic University, \\ Dohuk, Kurdistan Region-Iraq (Ammar.isam@dpu.edu.krd)
}

\begin{abstract}
:
This paper studies the optimization of the asymmetric magnetic recording heads, the calculations demonstrate sharp field gradient performance for both the horizontal, and vertical field components for this type of magnetic recording heads at large inclination and small distance between the head and underlayer. In addition, the characteristics for a higher bit density recording of HDD have been studied. These characteristics can be utilized to raise bit density in both recording method: conventional and vertical, respectively.
\end{abstract}

KEYWORDS: Magnetic Recording, Asymmetrical Heads, Potential and head field.

\section{INTRODUCTION}

The magnetic head can be considered as an integral part of magnetic recording system. The geometry and dimensions of any head will determine its fringing gap fields magnitude and distribution and its gradients as well, as a result it will affect the size and shape of the recorded magnetisation pattern in the magnetic medium during recording, and the resolving performance of the head in readout (Mee \& daniel, 1986) and (Bertram, 1994). This will lead to a standing fact that magnetic heads design has direct influence on the storage density achievement of magnetic recording systems (Mee \& daniel, 1986) and (Park et al., 2009).

The 2D magnetic recording heads, for the types of the ring-type, thin-film with finite-pole in addition to the single pole type head geometries have symmetrical pole geometry and with mostly symmetrical fringing fields producer in the gap area or in corner regions of the pole head (Suzuki \& Iwasaki, 1972) as illustrated in Fig 1 for the ring head geometry. On the other side, the asymmetric heads differ by rotating, in parallel, the pole corners in the gap region through exterior angle $\theta_{0}$ as indicated in 2D as illustrated in Fig. 2. On the $x$-axis is the direction along the head/medium motion, while the $y$-axis is normal to the head surface. This study will focus on the 2D geometry, the head depth along with the $z$ direction has been neglected.

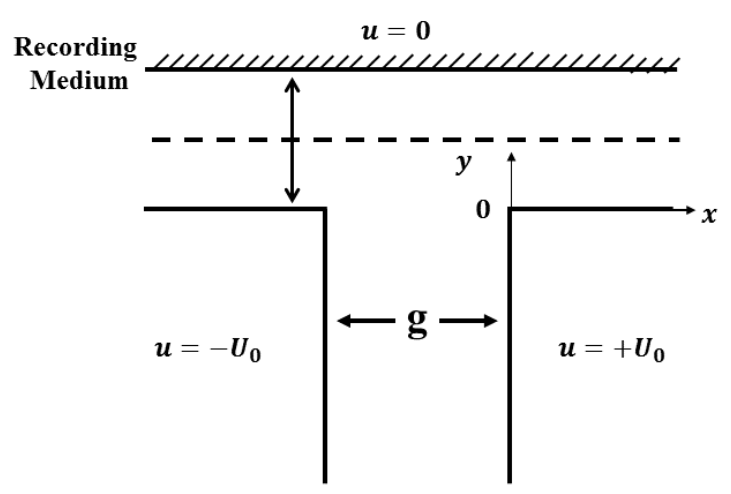

Fig. 1: PR head configuration, the ring head, with gap length g. The poles are assumed to have infinite permeability with potentials $\pm \mathrm{U}_{0}$ (Wilton, 1991).

The significance of the asymmetrical head shown in Fig. 2 arises from the increased magnetic charge density in the acute head corner (Jackson, 1999). This leads to an increase in the magnitude of the magnetic fields in this region and narrowing of their distributions as will be shown later in this paper. Asymmetric heads were therefore proposed for increasing the recording resolution in longitudinal and perpendicular recording due to the increased field gradients near the acute pole corner in the gap region (Wang et al., 1988). They were also suggested as a method of increasing the readout resolution of ring-type inductive heads and develop 'gapnull free' heads (Wang \& Huang, 1990), primarily through the natural reduction in the 'effective' gap length of the head with the increase in field enabling shorter recorded magnetization patterns in the recording medium to be resolved by the head. Asymmetric head designs were also incorporated in perpendicular heads 
with tapering in both the main pole and side shields to increase the recording fields and their gradients and to reduce side fringing (Wilton, 1991) and (Jackson, 1999). This tapered single-pole head structures with tapered shields, incorporating the asymmetric gapped geometry, were also investigated as part of corner-type head designs for high-resolution, two-dimensional magnetic recording (Victora et al., 2012).

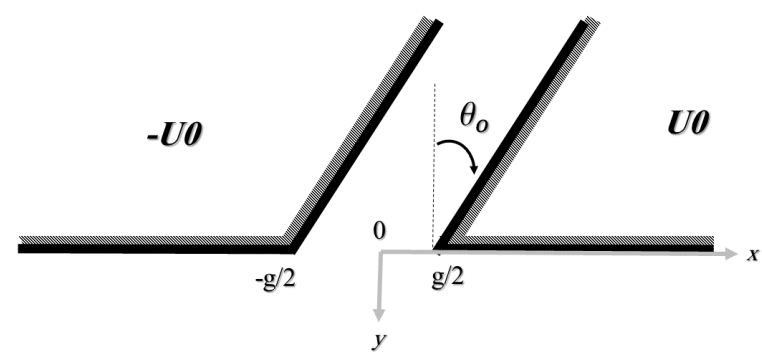

Fig. 2: Two-dimensional geometry of the asymmetrical head, with gap length $g$, exterior corner inclination angle $\theta o$ and poles potentials $\pm \mathrm{U}_{0}$ (Aziz et al., 2016).

The idea of validity of utilizing the conventional perpendicular magnetic recording to improve the areal density to reach up to $1 \mathrm{~Tb} / \mathrm{in} 2$, before upgrading to the heat assisted magnetic recording (HAMR) or patterned media is supported by many researchers (Wood, 2000), and (Batra et al., 2004). Because of the ultrahigh recording density produced by perpendicular magnetic recording, most research focuses on this method rather than, the conventional longitudinal magnetic recording. The ring-type head is employed along with the single layered media due to its easy implementation to the conventional hard disk technology (Batra et al., 2004).

The asymmetric ring head is considered as a good candidate to produce large field gradient and narrow field width, and hence overcoming the fundamental limitations to head field gradients (Wang et al., 1988). The considered geometry of the asymmetrical head is introduced previously in (Edress Mohamed, 2016) without underlayer, and when the underlayer is present in (Jackson, 1999), the core permeability of the considered head is assuming to be infinite. Also, the potential and field are derived for all possible practical inclination angles to serve the saturation effects to the mid of the operation of the head. In this work, the gradient of the vertical and horizontal field components for the asymmetrical head is evaluated and analyzed in order to demonstrate the quite suitable inclination angle for vertical recording purpose.

The analysis in both (Jackson, 1999) and (Edress Mohamed, 2016) show the characteristics of the near corner field for asymmetric heads when gap between the poles is tilted, that the Hx, and Hy field components were gradually departing from a symmetric to antisymmetric field distribution. It's also shows that, in case of increasing the inclination angle, the field is sharply peaked around the acute corner [see (Edress Mohamed, 2016) Fig. 3.6-3.10 for head without underlayer], and when the underlayer is present [See (Edress Mohamed, 2016) Fig. 4.2-4.6]. In this paper the result in (Edress Mohamed, 2016) and (Jackson, 1999) for the horizontal, and vertical field components behavior around the acute corner is shows a similar feature for the corresponding field gradient.

Although, a serious saturation problem could be accrued as a result of an increasing inclination angle on the acute comer, the aim of this work is to specify the optimum inclination angle for an appropriate performance for recording process and the optimum configuration of asymmetric ring head and hence, a high-density recording. This calculation will begin with finding the gradient of the horizontal components for 2D asymmetrical heads when SUL is absent, and then when a SUL is present.

\section{LONGITUDINAL RECORDING MODE}

The field gradient of the longitudinal mode for the asymmetrical recording head configuration for both cases are covered in this study in the absence of a SUL, and when the SUL is present is discussed next.

\section{Horizontal field gradient for asymmetrical head without} SUL.

The $\mathrm{x}$ field component for the asymmetrical head without underlayer is derived in (Gao \& Bertram, 2002) using the principle of superposition and given by the following equations:

$$
\begin{aligned}
H_{x}(x, y)= & \operatorname{Imag}\left(-A \alpha \frac{\sin (\alpha \pi)}{\pi} I_{\alpha}+B \beta \frac{\sin (\beta \pi)}{\pi} I_{\beta}\right) \\
& \ldots(1) \\
H_{y}(x, y)= & \text { Real }\left(-A \alpha \frac{\sin (\alpha \pi)}{\pi} I_{\alpha}+B \beta \frac{\sin (\beta \pi)}{\pi} I_{\beta}\right)
\end{aligned}
$$

where

$$
I_{\alpha}=\int_{-\frac{g}{2}}^{\frac{g}{2}} \frac{\left|x+\frac{g}{2}\right|^{\alpha-1}}{x^{\prime}-x-j y} d x^{\prime} \text {, and } I_{\beta}=\int_{-\frac{g}{2}}^{\frac{g}{2}} \frac{\left|x-\frac{g}{2}\right|^{\beta-1}}{x^{\prime}-x-j y} d x^{\prime}
$$

$I_{\alpha}$ and $I_{\beta}$ in (1) and (2) are evaluated using the HurwitzLerch Zeta function which is given in (Ferreira \& López, 2004):

$$
=\frac{1}{\Gamma(s)} \int_{0}^{\Phi(z, s, a)} \frac{t^{s-1} e^{-a t}}{1-z e^{-t}} d t
$$

where, $\Gamma(s)$ is the Gamma function. When $s=1$, the Zeta function becomes:

$$
\Phi(z, 1, a)=\int_{0}^{\infty} \frac{e^{-a t}}{1-z e^{-t}} d t
$$

The integral $I_{\alpha}$ in (4) can be evaluated by using the substitution $x^{\prime}=-t+\frac{g}{2}$ to yield: 


$$
I_{\alpha}=-\int_{0}^{g} \frac{t^{\alpha-1}}{t-z_{1}} d t
$$

where $z_{1}=\frac{g}{2}-x-j y$. Similarly, using the substitution $x^{\prime}=t-\frac{g}{2}$ to evaluate $I_{\beta}$ in (2) gives:

$$
I_{\beta}=\int_{0}^{g} \frac{t^{\beta-1}}{t-z_{2}} d t
$$

where $z_{2}=\frac{g}{2}+x+j y$.

The integrals $I \alpha$ and $I \beta$ were determined using Mathematica ${ }^{\circledR}$, and can be written in terms of the Hypergeometric function 2F1(Ferreira \& López, 2004) as:

$I_{\alpha}=-\frac{\left(g-z_{1}\right)^{\alpha}}{\alpha z_{1}}{ }_{2} F_{1}\left(-\alpha,-\alpha ; 1-\alpha, \frac{z_{1}}{z_{1}-g}\right)+\frac{g^{\alpha}}{\alpha z_{1}}-\left(-z_{1}\right)^{\alpha-1} \frac{\pi}{\sin (\alpha \pi)}$

$I_{\beta}=\frac{\left(g-z_{2}\right)^{\beta}}{\beta z_{2}}{ }_{2} F_{1}\left(-\beta,-\beta ; 1-\beta, \frac{z_{2}}{z_{2}-g}\right)-\frac{g^{\beta}}{\beta z_{2}}+\left(-z_{2}\right)^{\beta-1} \frac{\pi}{\sin (\beta \pi)}$

Equations (1) and (2) are used next to evaluate and plot the fields beyond the surface of asymmetrical heads.

The above equations are used to evaluate the $x$ field component for a range of angles. The shape of the left edge of the $H x$ component field, and the field gradient demonstrate that is, a great extent the bit density can achieve. Edress Mohamed in (Gao \& Bertram, 2002) plotted curves for the field gradients which can be obtained for three different angles $\left(\theta \mathrm{o}=0^{\circ}, 30^{\circ}, 45^{\circ}\right)$ at three different spacings from the head surface $(y / g=0.05$, and 0.1 ) as shown in Fig. 3:

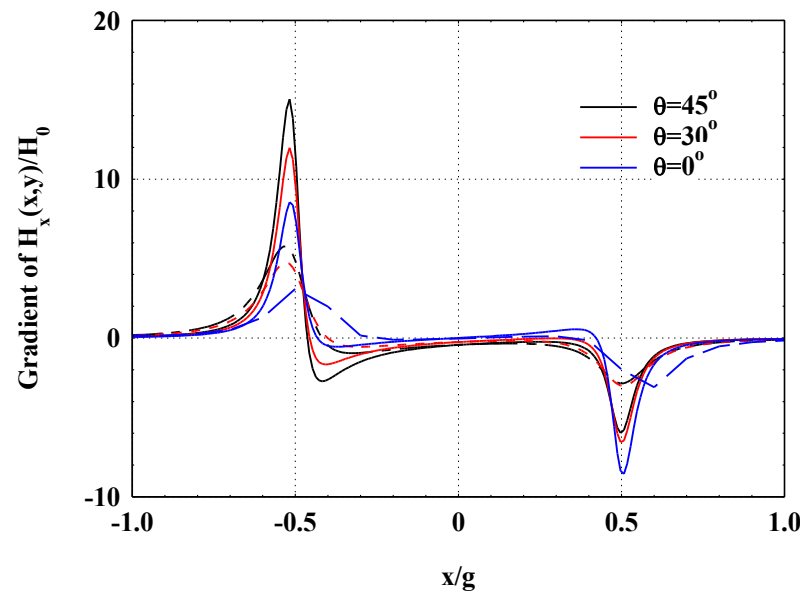

Fig. 3: x-component field gradient distribution for the asymmetrical head without underlayer for interior corner angle $0 \mathrm{o} \leq \theta \mathrm{o} \leq 45 \mathrm{o}$ at $\mathrm{y} / \mathrm{g}=0.05$ (solid lines), and $\mathrm{y} / \mathrm{g}=0.1$ (dashed lines).
The Table 1, illustrates the maximum gradient values for each inclination angles at different spacings from the head surface.

TABle 1: THE MAXIMUM GRADIENT VALUES OF $H x$ FIELD FOR ASYMMETRICAL HEAD WITHOUT SUL FOR $0^{\circ} \leq \theta_{\mathrm{o}} \leq 45^{\circ}$ AT $\mathrm{y} / \mathrm{g}=0.05$, 0.1, AND 0.5

\begin{tabular}{|c|c|c|c|c|}
\cline { 2 - 5 } \multicolumn{1}{c|}{} & $\boldsymbol{\theta}_{\mathbf{o}}$ Angle & $\mathbf{0}^{\mathbf{o}}$ & $\mathbf{3 0}^{\mathbf{o}}$ & $\mathbf{4 5}^{\mathbf{0}}$ \\
\hline \multirow{3}{*}{ Field gradient } & $\boldsymbol{y} / \boldsymbol{g}=\mathbf{0 . 0 5}$ & 8.56 & 11.98 & 15.03 \\
\cline { 2 - 5 } & $\boldsymbol{y} / \boldsymbol{g}=\mathbf{0 . 1}$ & 3.09 & 4.76 & 5.78 \\
\cline { 2 - 5 } & $\boldsymbol{y} / \boldsymbol{g}=\mathbf{0 . 5}$ & 0.98 & 1.08 & 0.62 \\
\hline
\end{tabular}

Table 1 shows for $y / g=0.05$, and 0.1 that, at the larger inclination, the greater the field gradient, and the higher the bit density it can achieve. For $y / g=0.5$ the greatest field gradient is recorded at $\theta \mathrm{o}=30^{\circ}$.

Horizontal field gradient for asymmetrical head with underlayer

For the case when soft underlayer is added to the asymmetrical head, the horizontal field component is discussed in detail and evaluated in (Gao \& Bertram, 2002) and (Wang et al., 19888)using the surface potential of the asymmetrical head without underlayer, along with its appropriate green function which defined as:

$$
u(x, y)=\frac{1}{2 d} \sin \left(\frac{\pi y}{d}\right) \int_{-\infty}^{\infty} \frac{u(\dot{x}, 0)}{\cosh \left(\frac{(\dot{x}-x) \pi}{d}\right)-\cos \left(\frac{\pi y}{d}\right)} d \dot{x} . .
$$

for $0<y<d$

For a range of inclination angles at $y / g=0.1$ as a spacing from the head surface where the underlayer is located at $g / d=0.25,0.5$, and 1 . The shape of the right edge of the $H_{x}$ component field, and the field gradient shows less amplitude comparing with the case of head without underlayer. The field gradients can be obtained for different 3 angles $\left(\theta_{o}=0^{\circ}, 30^{\circ}, 45^{\circ}\right)$ at $y / g=0.1$ from the head surface as shown in Fig. 4 :

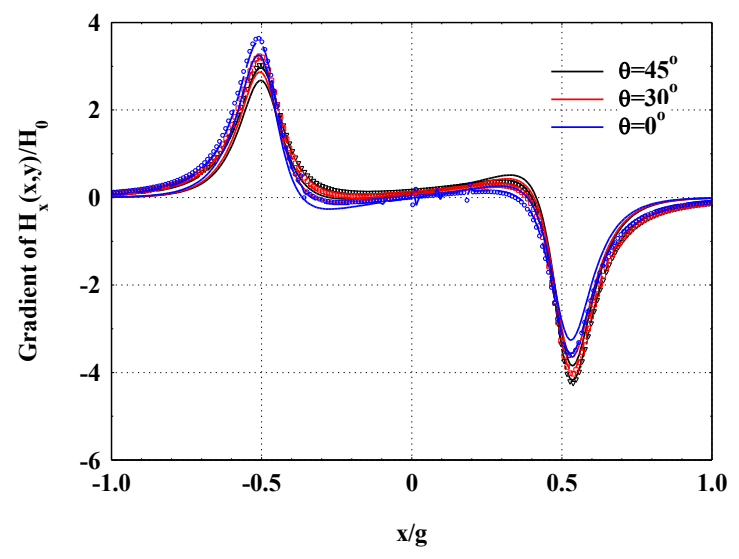

Fig. 4: $\mathrm{x}$-component field gradient distribution for the asymmetrical head with underlayer for interior corner angle $0^{\circ}$ $\leq \theta_{\mathrm{o}} \leq 45^{\circ}$ at head surface-to-SUL distance $\mathrm{g} / \mathrm{d}=0.25$ (solid lines), $\mathrm{g} / \mathrm{d}=0.5$ (dashed lines), $\mathrm{g} / \mathrm{d}=1$ (doted lines).

The Table 2, shows the maximum gradient values for each inclination angles at $y / g=0.1$ when the soft underlayer is located at $g / d=0.25,0.5$, and 1 from the head surface. 
TABLE 2: THE MAXIMUM GRADIENT VALUES OF HX FIELD GRADIENT FOR ASYMMETRICAL HEAD WITH SUL $0^{\circ} \leq \theta_{\mathrm{O}} \leq 45^{\circ}$ AT $\mathrm{y} / \mathrm{g}=0.1$ FOR $\mathrm{g} / \mathrm{d}=0.25,0.5$, AND 1

\begin{tabular}{|c|c|c|c|c|}
\cline { 2 - 5 } \multicolumn{1}{c|}{} & $\boldsymbol{\theta}_{\mathbf{o}}$ Angle & $\mathbf{0}^{\mathbf{0}}$ & $\mathbf{3 0}^{\mathbf{0}}$ & $\mathbf{4 5}^{\mathbf{0}}$ \\
\hline \multirow{4}{*}{$\begin{array}{c}\text { Field } \\
\text { gradient }\end{array}$} & $\boldsymbol{g} / \boldsymbol{d}=\mathbf{0 . 2 5}$ & 3.26 & 2.87 & 2.67 \\
\cline { 2 - 5 } & $\boldsymbol{g} / \boldsymbol{d}=\mathbf{0 . 5}$ & 3.56 & 3.16 & 2.95 \\
\cline { 2 - 5 } & $\boldsymbol{g} / \boldsymbol{d}=\mathbf{1}$ & 3.62 & 3.22 & 3.02 \\
\hline
\end{tabular}

Table 2 shows for all $y / g$, at the smaller inclination, the greater the field gradient, and the higher the bit density it can achieve.

\section{PERPENDICULAR RECORDING MODE}

The field gradient of the vertical mode for the asymmetrical recording head geometry when absence of a soft underlayer and when the underlayer is present is discussed next.

\subsection{Vertical field gradient for asymmetrical head without underlayer}

The vertical field gradient is derived in (Gao \& Bertram, 2002) using the principle of superposition and given by the equation (2), which is utilized to evaluate the $y$ field component for a range of angles. The usefulness of the head is determined by the vertical component field distribution, as illustrated in (Gao \& Bertram, 2002) (see Fig.s 3.6-3.9). The greatest field gradient starts to occur at the inner side of the sharp edge of the head.

The shape of the left edge of the $H_{y}$ component field, and the field gradient demonstrate that is, a great extent the bit density it can achieve. The field gradients can be obtained for different 3 angles $\left(\theta \mathrm{o}=0^{\circ}, 30^{\circ}, 45^{\circ}\right)$ at three different spacings from the head surface $(y / g=0.05,0.1$, and 0.5) as shown in Fig. 5:

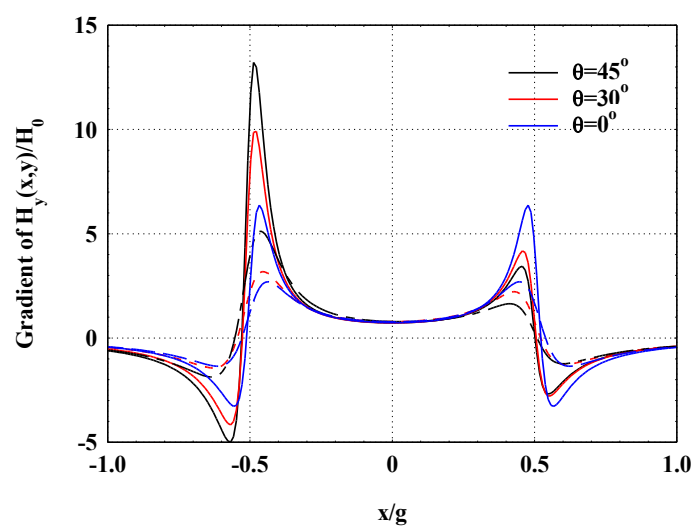

Fig. 5: y-component field gradient distribution for the asymmetrical head without underlayer for interior corner angle $0^{\circ} \leq \theta_{0} \leq 45^{\circ}$ at $\mathrm{y} / \mathrm{g}=0.05$ (solid lines), and $\mathrm{y} / \mathrm{g}=0.1$ (dashed lines).

Table 3 shows the maximum y-component field gradient values for a range of inclination angles.

Table 3: The Maximum Gradient Values Of Hy Field Gradient For Asymmetrical Head Without SUL 0o $\leqslant$

\begin{tabular}{|c|c|c|c|c|}
\hline & $\theta_{0}$ Angle & $0^{\circ}$ & $30^{\circ}$ & $45^{\circ}$ \\
\hline \multirow{3}{*}{$\begin{array}{c}\text { Hy } \\
\text { Field } \\
\text { gradient }\end{array}$} & $y / g=0.05$ & 6.36 & 9.90 & 13.21 \\
\hline & $y / g=0.1$ & 2.7 & 3.18 & 5.11 \\
\hline & $y / g=0.5$ & 0.55 & 0.56 & 0.69 \\
\hline
\end{tabular}

Fig. 5, supported by Table 3 demonstrate that for all $y / g$, at a greater inclination angle, the greater the field gradient, and the higher the bit density it can achieve. Hence, we can say that the $\theta 0=45^{\circ}$ has a higher field gradient.

\subsection{Vertical field gradient for asymmetrical head with} underlayer

The $y$ field component for the asymmetrical head when the soft underlayer is present and located at $g / d$ from the head surface is derived in (Gao \& Bertram, 2002) using two different techniques. The surface potential for the asymmetrical head with underlayer is used convolving with the Green function as defined in equation (9).

The gradient of the vertical field component for the asymmetrical head without underlayer is plotted using the equation (2). Fig. 6 shows the gradient values for a range of angles at three spacing from the head surface $(g / d=0.25,0.5$, and 1$)$.

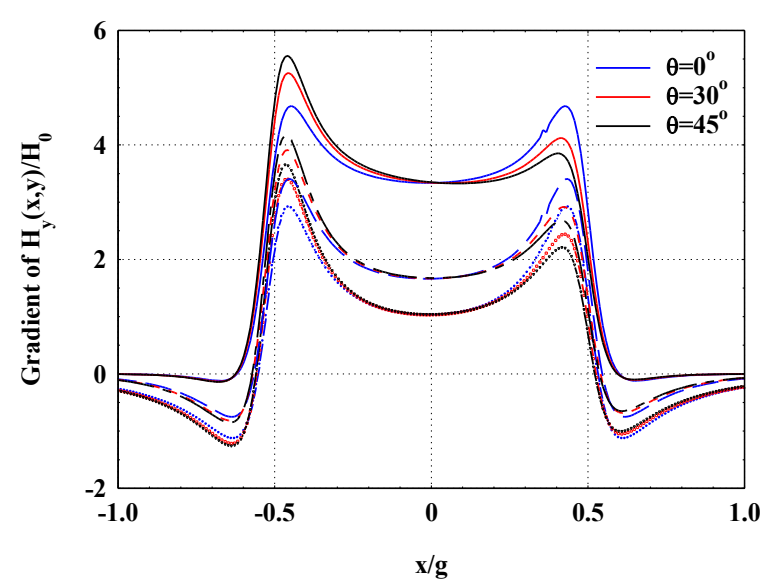

Fig. 6: y-component field gradient distribution for the asymmetrical head with SUL for interior corner angle $0^{\circ} \leq \theta_{0} \leq$ $45^{\circ}$ at head surface-to-SUL distance $\mathrm{g} / \mathrm{d}=0.25$ (solid lines), $\mathrm{g} / \mathrm{d}=0.5$ (dashed lines), $\mathrm{g} / \mathrm{d}=1$ (doted lines).

Table 4, illustrates the gradient values for each inclination angles at different spacings from the head surface.

Table 4: The maximum gradient values of Hy field gradient for asymmetrical head with SUL for $0 \mathrm{o} \leq \theta \mathrm{o} \leq 45 \mathrm{o}$ at $\mathrm{y} / \mathrm{g}=0.1$ for $\mathrm{g} / \mathrm{d}=0.25,0.5$, and 1

\begin{tabular}{|c|c|c|c|c|}
\hline \multirow{2}{*}{$\begin{array}{c}\text { Hy } \\
\text { Field } \\
\text { gradient }\end{array}$} & $\boldsymbol{\theta}_{\mathbf{o}}$ Angle & $\mathbf{0}^{\mathbf{0}}$ & $\mathbf{3 0}^{\mathbf{0}}$ & $\mathbf{4 5}^{\mathbf{0}}$ \\
\cline { 2 - 5 } & $\boldsymbol{g} / \boldsymbol{d}=\mathbf{0 . 2 5}$ & 4.67 & 3.40 & 2.91 \\
\cline { 2 - 5 } & $\boldsymbol{g} / \boldsymbol{d}=\mathbf{0 . 5}$ & 5.26 & 3.91 & 3.39 \\
\cline { 2 - 5 } & $\boldsymbol{g} / \boldsymbol{d}=\mathbf{1}$ & 5.55 & 4.16 & 3.65 \\
\hline
\end{tabular}

Fig. 6, and Table 4 show that for all $\mathrm{y} / \mathrm{g}$ that, at the larger inclination, the greater the field gradient, and the higher the bit density it can achieve.

\section{CONCLUSION}

Here we have determined that in case of increasing of inclination angle of an asymmetric heads the characteristics which is required for high bit density recording than is possible for non-inclined heads configuration will improve. The asymmetric heads for the case when the SUL is present demonstrated a soft underlayer double the head field comparing with the asymmetric heads without underlayer. 


\section{REFERENCES}

A. Edress Mohamed, "Theory of Symmetry and Asymmetry In Two- Dimensional Magnetic Recording Heads," University of Exeter, 2016.

C. D. Mee and E. D. Daniel, Magnetic Recording; Vol. I: Technology. New York, NY, USA: McGraw-Hill, Inc., 1986.

C. Ferreira and J. L. López, "Asymptotic expansions of the Hurwitz-Lerch zeta function," J. Math. Anal. Appl., vol. 298, no. 1, pp. 210-224, Oct. 2004.

C. S. Wang and H. L. Huang, "Gap-null free spectral response of asymmetric ring heads for longitudinal and perpendicular recording," Magnetics Conference, 1990. Digests of INTERMAG '90. International. p. FP-FP, 1990.

C. S. Wang, H. L. Huang, Chia Shen Wang, and Huei Li Huang, "Characteristics Of Asymmetric Heads," J. Phys., vol. 49, no. 12, pp. 2023-2024, 1988.

D. T. Wilton, "An analysis of the magnetic field of a ring head with a highly permeable underlayer," IEEE Trans. Magn., vol. 27, no. 4, pp. 3751-3755, 1991.

H. N. Bertram, Theory of Magnetic Recording, 1st ed. New York: Cambridge University Press, 1994.

J. D. Jackson, Classical Electrodynamics Third Edition, 3rd ed. United State -NY: Library of Congress Cataloging-inOublication Data, 1999.

K. Z. Gao and H. N. Bertram, "Magnetic recording configuration for densities beyond $1 \mathrm{~Tb} / \mathrm{in} 2$ and data rates beyond $1 \mathrm{~Gb} / \mathrm{s}$," IEEE Trans. Magn., vol. 38, no. 6, pp. 3675-3683, 2002.

M. M. Aziz, A. I. Edress, and C. D. Wright, "Approximate Expressions for the Magnetic Potential and Fields of 2-D, Asymmetrical Magnetic Recording Heads," IEEE Transactions on Magnetics, vol. 52, no. 2. pp. $1-12,2016$.

R. H. Victora, S. M. Morgan, K. Momsen, E. Cho, and M. F. Erden, "Two-Dimensional Magnetic Recording at 10 Tbits/in2," IEEE Trans. Magn., vol. 48, no. 5, pp. $1697-1703,2012$

R. Wood, "The feasibility of magnetic recording at 1 Terabit per square inch," IEEE Trans. Magn., vol. 36, no. 1, pp. $36-42,2000$

S. Batra, J. D. Hannay, H. Zhou, and J. S. Goldberg, "Investigations of Perpendicular Write Head Design for $1 \mathrm{~Tb} / \mathrm{in}^{\wedge} 2$," IEEE Trans. Magn., vol. 40, no. 1, pp. 319-325, 2004.

S. P. S. Park, J. Y. J. Yoo, and J. S. C. J. S. Choi, “Optimal Shape Design of the Perpendicular Magnetic Recording Head," IEEE Trans. Magn., vol. 45, no. 5, pp. 22722275, 2009.

T. Suzuki and S.-I. Iwasaki, "An Analysis of Magnetic Recording Head fields Using a Vector Potential," IEEE Trans. Magn., no. September, pp. 536-538, 1972. 